\title{
EVALUACIÓN DE LA SUSTENTABILIDAD DEL SISTEMA Milpa En El estado de TlaXCala, MÉXico
}

\author{
Evaluation of sustainability of the milpa system \\ in the Tlaxcala State, Mexico
}

PRiMO SÁNCHEZ MORALES*

OMAR ROMERO ARENAS**

\section{RESUMEN}

El objetivo del estudio es efectuar una evaluación comparativa de la sustentabilidad de dos sistema de producción: a) sistema milpa tradicional y b) sistema de maíz en monocultivo. Para conocer las fortalezas y las debilidades se calculó el tamaño de la muestra $(n=380)$ a partir de la población de productores de maíz, $\mathrm{N}=29$ 828. La metodología fue construida desde una perspectiva agroecológica: se aplicó el Marco para la Evaluación de Sistemas de Manejo de Recursos Naturales incorporando indicadores de sustentabilidad y se consideró la escala temporal transversal en el ciclo primavera-verano 2014 en el estado de Tlaxcala, México. Se establecieron 15 indicadores, en los que se abarcaron las dimensiones social, económica y ambiental. Los resultados muestran que solo en un indicador económico, el sistema monocultivo obtuvo el valor más alto; en dos indicadores los valores son similares (balance oferta/demanda de grano y zacate e impacto de políticas agrícolas); en el resto, los resultados favorecieron al sistema milpa. En tres de estos, la diferencia es mayor a 35 puntos porcentuales: relación beneficio/costo, eficiencia en uso de energía fósil y agrodiversidad. Se concluye que en el sistema milpa es favorable la asociación de cultivos, se aprovecha de mejor manera el espacio y los energéticos fósiles empleados y se produce mayor diversidad de alimento humano. Sin embargo, hace falta integrar otros cultivos y prácticas que hagan más sustentables ambos sistemas de producción.

Palabras Clave: maíz, policultivo, monocultivo, indicadores, MESMis.

* Benemérita Universidad Autónoma de Puebla, Centro de Agroecología del Instituto de Ciencias. Correo electrónico: primosamo@yahoo.com.

** Benemérita Universidad Autónoma de Puebla, Centro de Agroecología del Instituto de Ciencias. Correo electrónico: biol.ora@hotmail.com. 


\section{Abstract}

The aim of this study is to evaluate the sustainability of comparative way between two corn production systems: (a) traditional milpa system, and (b) system of maize in monoculture. For strengths and weaknesses we calculated the sample size $(n=380)$ from the population of corn producers $\mathrm{N}=29.828$. The methodology was built from an agroecological perspective; applied the Framework for the Assessment of systems of Natural Resource Management incorporating sustainability indicators, and considered the time scale cross-spring-summer 2014 in the state of Tlaxcala, Mexico. 15 indicators were established in which covered the dimensions: social, economic and environmental. The results show that only in an economic indicator, the system monoculture obtained higher value; in two indicators values are similar (supply/demand balance of grain and grass, and impact of agricultural policies); in the remainder, the results favored the milpa system. In three of these, the difference is more than 35 percentage points: cost-benefit ratio, efficiency in use of fossil energy, and agrobiodiversity. It is concluded that in the milpa is favorable the association of crops and takes advantage of best way the space and fossil fuels employees and produces greater diversity of human food; however, there is a need to integrate other crops and practices that make both more sustainable production systems.

KEYWORDS: CORN, POLYCULTURE, MONOCULTURE, INDICATORS, MESMIS.

Recepción: 4 de marzo de 2016.

Dictamen 1: 11 de mayo de 2016.

Dictamen 2: 22 de noviembre de 2016. 


\section{INTRODUCCIÓN}

El maíz es el grano que más se cultiva y consume en el mundo. De acuerdo con el Departamento de Agricultura de Estados Unidos (USDA), en 2014 se estimó la cosecha en 1006.24 millones de toneladas métricas (TM) (USDA, 2015). Estados Unidos es el mayor productor y el principal consumidor de maíz. Sin embargo, el consumo en este país no ocurre de manera directa: primero es transformado en carne, leche y huevo. Además, se calcula que en 2011 en Estados Unidos se sembró 82 por ciento de la superficie de maíz con semillas transgénicas (González y Ávila, 2014; USDA, 2014).

En el caso de México, el consumo es directo. Se calcula un consumo per cápita de 343 gramos (CEDRSSA, 2014) y se importan alrededor de 10 millones de TM. De acuerdo con Hernández Xolocotzi, desde su propuesta etnobotánica y de "la agricultura de huarache", este grano es originario de México, y se cultiva desde hace aproximadamente nueve mil años (Hernández y Aguirre, 1998, pp. 104-109). Las formas de cultivo se valieron de tecnologías de bajos insumos, fueron de poca dependencia externa y, en general, de baja entropía (Max Neef et al., 2001, pp. 26-28).

No obstante, en las décadas recientes se han implementado técnicas "productivistas" como el paquete de la revolución verde (RV), que desde la década de los sesenta del siglo XX ha tenido el objetivo de incrementar los rendimientos por área sembrada mediante el uso de semillas híbridas, fertilización química, maquinaria y equipo con alto uso de energéticos fósiles. En la actualidad se integró la propuesta de utilizar semillas transgénicas, que lo hace cada vez menos sustentable y con una entropía alta (Max Neefet al., 2001, pp. 26-28), lo cual atenta contra el ambiente, la agrodiversidad y la cultura de los pueblos indígenas y campesinos (Hecht, 1999, pp. 28-29).

En el presente predomina el cultivo de maíz en monocultivo, con algunas desventajas como la pérdida de especies; el deterioro de los suelos por la extracción de nutrientes similares en cada ciclo y por erosión a causa de las prácticas "modernas"; la vulnerabilidad de los agroecosistemas ocasionada por la reducción de la base genética; la alta dependencia de energéticos fósiles, y la disminución de especies dentro de las cadenas tróficas provocada por el uso de pesticidas, entre otros (Hecht, 1999, pp. 28-29).

En Mesoamérica, antes de la RV, el maíz se sembraba de forma tradicional, generalmente en policultivo con frijol, calabaza y chile, entre otros (sistema milpa), lo que significó una producción más diversificada de alimentos y, a la vez, un menor desgaste de nutrientes en los suelos. El sistema milpa se considera una alternativa 
viable que combina ingeniosas prácticas campesinas e indígenas, que integra productos para la diversificación de la dieta. Además, la Food and Agriculture Organization (FAO) lo reconoce como un "sistema importante del patrimonio agrícola mundial” (FAO, 2009). El sistema milpa es una de las diversas formas de conservación del germoplasma nativo. De acuerdo con De Schutter (2012, pp. 15-19), se debe proteger legalmente el manejo de la milpa y mejorar su potencial productivo porque es un mecanismo para abatir la pobreza alimentaria.

Ante las consideraciones citadas y la proporción de productores mexicanos de subsistencia, el objetivo general de esta investigación es conocer la sustentabilidad de los sistemas milpa tradicional y cultivo de maíz en monocultivo, para conocer los saberes campesinos que aportan a la revaloración de este policultivo tradicional y contribuir con elementos teóricos para el estado de Tlaxcala, México.

\section{MATERIALES Y MÉTODOS}

\section{Ubicación geográfica}

El estado de Tlaxcala es la entidad más pequeña de la república mexicana, con una extensión de 3997 kilómetros cuadrados. El clima es templado subhúmedo C(w) en 92 por ciento de su extensión geográfica; la temperatura media anual es de 14.5 grados centígrados. La precipitación media anual es de 720 milímetros, con lluvias en verano, principalmente entre los meses de julio a diciembre (SAGARPA, 2014).

Tlaxcala se ubica en el altiplano central mexicano, entre los $19^{\circ} 06^{\prime} 18^{\prime \prime}-19^{\circ}$ $43^{\prime} 44^{\prime \prime}$ de latitud norte y entre los $97^{\circ} 37^{\prime} 32^{\prime \prime}$ - 98 42'31" de longitud oeste, a una altitud media de 2230 metros sobre el nivel del mar (INEGI, 2015). Al suroeste, sur, este y una sección del norte colinda con el estado de Puebla; al norte y noroeste, con el estado de Hidalgo, y en una pequeña franja del noroeste comparte límites con el Estado de México. La entidad está dividida en 60 municipios. De acuerdo con el Censo de Población y Vivienda, en 2015 tenía 1272847 habitantes, de los cuales 80 por ciento vive en zonas urbanas. Según el Instituto Nacional de Estadística y Geografía (INEGI, 2016), las zonas urbanas son comunidades con más de 2500 habitantes.

La escolaridad promedio de la población tlaxcalteca en el periodo 2013-2014 fue de 9.1 años, ligeramente superior a la media nacional, de 9.0; mientras que el índice de analfabetismo es de 4.3 por ciento, inferior al índice nacional, de 6.0 por ciento (SE, 2015). 
Los principales cultivos, en orden de importancia, son: maíz (115 503 hectáreas), cebada (42 898 hectáreas) trigo (33 519 hectáreas), frijol (5 231 hectáreas), haba (4 176 hectáreas) (SIAP, 2014). Los suelos que predominan son: Phaeozem 34.2 por ciento), Regosol (13.6 por ciento), Durisol (12.0 por ciento), Leptosol (11.6 por ciento) (INEGI, 2012). Los suelos tienen una profundidad menor a un metro en la mayor parte del estado, por lo cual se consideran someros. Además, existe algún grado de erosión en más de 90 por ciento del territorio estatal (INE, 1995).

\section{Recolección de datos}

Para la colecta de información se consideró el universo de beneficiarios del programa de subsidio gubernamental federal Proagro Productivo de 2014 para el estado de Tlaxcala. Los beneficiarios reportados fueron en total 43219 campesinos, de los cuales 29828 son productores de maíz (Proagro Productivo, 2014).

Se dividió el estado de Tlaxcala en cuatro regiones geográficas: norte, oriente, sur y poniente, con el objetivo de distribuir proporcionalmente los productores que se entrevistarían en cada región. Se calculó el tamaño de la muestra (Hernández et al., 2010, pp. 170-190) y, por afijación proporcional, se determinó la equivalencia para cada uno de los estratos (regiones) (Cochran, 1974; Azorín, 1969). El muestreo fue aleatorio simple con varianza máxima, precisión de cinco por ciento y el valor de la distribución normal de 1.96, con probabilidad de error de cinco por ciento y nivel de confianza de 95 por ciento. Con estas variables descritas, la fórmula que se utilizó para calcular el tamaño de la muestra fue la siguiente:

Al sustituir los valores de las variables involucradas, donde $\mathrm{N}=29828$ productores, resultó el valor de $n=380$. Posteriormente se aplicó la afijación proporcional para cada una de las regiones, lo cual determinó el número de productores que siembran maíz en cada región, que quedaron distribuidos de la siguiente manera: 1) norte $\mathrm{n}_{1}=87 ; 2$ ) oriente $\mathrm{n}_{2}=165 ; 3$ ) sur $\mathrm{n}_{3}=64, \mathrm{y} 4$ ) poniente $\mathrm{n}_{4}=64$. El estado de Tlaxcala tiene condiciones diferidas en las cuatro regiones, por ejemplo, el tamaño de los predios que poseen y siembran los productores y los cultivos más importantes de cada región. Se aplicó la encuesta a un total de 380 productores, que se eligieron al azar de la lista de beneficiarios de Proagro Productivo.

Para evaluar la sustentabilidad, se utilizó el marco para la evaluación de sistemas de manejo de recursos naturales incorporando indicadores de sustentabilidad (MESMIS), que propone seis pasos básicos (Masera et al., 2000, p. 27). Se aplicó la encuesta a los agricultores distribuidos en 53 municipios de la entidad tlaxcalteca 
(los siete municipios restantes tienen pocos campesinos beneficiarios de Proagro Productivo, razón por la que no salió sorteado alguno de ellos). Los resultados se ordenaron y codificaron en una base de datos del programa Excel para Windows, lo cual permitió clasificar el total de encuestados en dos grandes grupos de productores de acuerdo con los propósitos de este trabajo: a) sistema milpa tradicional (SMT), y b) sistema de maíz en monocultivo (SMo).

Una vez clasificados de la manera antes mencionada, se realizaron algunas pruebas de validación a la base de datos de cada sistema como lo sugieren Hernández et al. (2010, p. 297). Posteriormente se realizaron los análisis en el programa Statistical Package for the Social Sciences (SPSS) versión 16, se determinaron resultados de estadística descriptiva, así como otros requerimientos para la valoración de los indicadores planteados en la evaluación comparativa de la sustentabilidad entre ambos sistemas de producción.

\section{Enfoque metodológico}

El enfoque teórico fue el agroecológico, cuyo fin es analizar los procesos agrícolas y entender la problemática de forma integral y holística. Por lo tanto, se habla de un paradigma cuyo enfoque considera los agroecosistemas como las unidades fundamentales de estudio; en estos, los ciclos minerales, las transformaciones de la energía, los procesos biológicos y las relaciones socioeconómicas son analizados e investigados como un todo (Altieri, 1987, pp. 31-33). Además, Sevilla y González (1992) consideran la agroecología como el manejo ecológico de los recursos naturales a través de formas de acción social-colectiva.

La perspectiva de este trabajo es transversal (ciclo de cultivo primavera-verano/2014) y, a la vez, tridimensional: se consideraron factores sociales, económicos y ambientales, (Altieri y Nicholls, 2000, p. 22). La escala espacial fue el estado de Tlaxcala. La estrategia teórica y metodológica tiene una naturaleza sistémica y un enfoque holista. El enfoque metodológico aquí empleado es de análisis comparativo, que involucra la comparación de un sistema de producción en monocultivo (SMo) con un sistema de producción de mayor complejidad (SMT) (Hecht, 1999, pp. 26-27), que para este caso es un policultivo tradicional mesoamericano. 


\section{RESULTADOS Y DISCUSIÓN}

\section{Caracterización de los sistemas de producción}

Se caracterizaron los dos sistemas de producción, los cuales tienen diferencias marcadas, que se mencionan a continuación:

a) Sistema milpa tradicional (SMT)

A este sistema también le llamaremos "sistema de referencia" por ser el sistema tradicional. Este sistema de producción es resultado de la conservación de prácticas tradicionales de indígenas y campesinos de Mesoamérica. De acuerdo con la zona, recibe diversos nombres; por ejemplo, en la zona de Yucatán, México, lo llaman "solar maya"; mientras que en diversos estados como Chiapas y Tlaxcala, entre otros, llaman "milpa" o "sistema milpa tradicional" a la asociación de maíz (gramínea), frijol (leguminosa) y calabaza (cucurbitácea) (Sánchez y Castro, 2011, pp. 76-79; Sánchez y Hernández, 2014, pp. 6-7).

Este policultivo tiene diversas variantes en función de las condiciones biofísicas: en el caso de Tlaxcala, en lugares con altitudes mayores a 2500 metros sobre el nivel del mar, el frijol (Phaseolus vulgaris L.) es sustituido por variedades de ayocote (Phaseolus coccineus L.) o haba (Vicia faba). También se han encontrado campesinos que solo siembran maíz-frijol, maíz-haba o maíz-calabaza. No obstante, para efectos de este trabajo, se consideran productores del SMT los que asocian maíz con alguna leguminosa, debido a la complementariedad en el sistema de cultivo y a la satisfacción de requerimiento de alimentos para sus familias.

En el SMT se ubicó a 20 por ciento del total de productores encuestados, cuyas edades varían de 35 a 86 años (con media y mediana de 58 años); poseen en promedio 3.7 hectáreas, aunque el rango varía de 0.5 hasta 9.0 hectáreas. El grupo de encuestados está conformado por 14 por ciento de mujeres y el resto de hombres. En general, la escolaridad media es cercana a cinco años, y 6.8 por ciento del total no sabe leer ni escribir. Además, 28 por ciento de los productores tiene otro trabajo para la generación de ingresos complementario a las actividades primarias. En promedio, el número de integrantes por familia es de cinco personas, aunque 57 por ciento de las familias se conforma de entre dos a cuatro y el resto lo integran de cinco a 11 personas.

b) Sistema de maíz en monocultivo (SMo)

En este trabajo nos referiremos a este sistema también como sistema alternativo, debido a que es un sistema industrial. Es el sistema de producción en el que solo se 
siembra maíz. Esto permite que se mecanice y se realicen aspersiones de herbicidas sin el riesgo de eliminar cultivos de hoja ancha. Esta forma de cultivo es resultado de la difusión de más de cinco décadas del paquete tecnológico de la revolución verde, implementado en México desde la década de los sesenta del siglo XX con el objetivo de industrializar la agricultura (Hewitt, 1985, pp. 47, 56).

En el SMo se ubica 80 por ciento de productores muestreados en el estado de Tlaxcala, con una edad media de 62 años y un rango que va de 23 a 93 años. La cantidad de tierra que poseen en promedio es de 5.5 hectáreas; sin embargo, el rango entre los que menos tienen y los que más área poseen va de 0.5 a 60.0 hectáreas. En este conjunto, 15 por ciento de las personas encuestadas son mujeres, proporción similar al primer grupo. La escolaridad también es similar a la del SMT, aunque el nivel de analfabetismo es ligeramente menor en este conjunto (6.2 por ciento). El número de integrantes por familia también es de cinco personas; 68 por ciento está compuesto de dos a cinco integrantes y el resto desde seis hasta 14. Además, 30 por ciento de los productores tiene algún oficio extra que les reporta ingresos complementarios para el mantenimiento de sus familias.

\section{Resultados en la aplicación del MESMIS}

El área de estudio representa 2.0 por ciento de la destinada a la producción de maíz en Tlaxcala. Sin embargo, con el agrupamiento realizado, el SMo conforma cerca de 1.8 por ciento, debido a que el maíz se cultiva de esta manera comúnmente. Para la evaluación de la sustentabilidad a través del MESMIS, se siguieron seis pasos sugeridos por Masera et al. (2000, pp. 5-7), como a continuación se citan.

Definición del objeto de la evaluación (paso 1). Debido a que ya hemos definido antes el objeto de este trabajo, nos permitimos continuar con el siguiente paso sugerido por Masera et al. (2000, pp. 32, 40).

Identificación de los puntos críticos del sistema (paso 2). De acuerdo con el análisis de los aspectos que limitan o fortalecen la capacidad de los sistemas para sostenerse a través del tiempo en la producción de maíz en Tlaxcala, los resultados de esta fase muestran que para los técnicos y los productores existen diversas consideraciones: algunos puntos críticos están relacionados con la productividad del agroecosistema (baja producción, alto consumo de combustibles, etcétera); otros son afines a la estabilidad, como el caso del monocultivo y la degradación de recursos naturales. Además, factores como el bajo precio del maíz, lo poco redituable que 
resulta este cultivo por la alta dependencia al paquete de la RV, o el riesgo de las heladas, se integraron al resto de atributos (véase el cuadro 1).

CuAdro 1. Puntos CRÍTICOS DEL SISTEMA DE PRODUCCiÓN MAÍZ

\begin{tabular}{ll}
\hline \multicolumn{1}{c}{ Atributos } & \multicolumn{1}{c}{ Puntos críticos } \\
\hline Productividad & $\begin{array}{l}\text { Baja producción, baja disponibilidad de forraje en algunas épocas del año, } \\
\text { bajo aseguramiento del autoconsumo, bajo valor agregado, alto consumo de } \\
\text { combustibles. }\end{array}$ \\
\hline Estabilidad, resiliencia y confiabilidad & $\begin{array}{l}\text { Monocultivo, interés decreciente hacia la agricultura por parte de las nuevas } \\
\text { generaciones, recursos naturales degradados. }\end{array}$ \\
\hline Adaptabilidad & $\begin{array}{l}\text { Precios bajos del maíz, aspectos climatológicos poco predecibles (heladas, } \\
\text { sequías); alto costo de insumos externos (insumos agrícolas y combustibles). }\end{array}$ \\
\hline Equidad & $\begin{array}{l}\text { Baja rentabilidad, limitado abasto de granos, acaparamiento por intermedia- } \\
\text { rios (coyotes). }\end{array}$ \\
\hline Autodependencia (autogestión) & $\begin{array}{l}\text { Alta dependencia de insumos externos, mucha mano de obra requerida, } \\
\text { falta de empleo bien remunerado y alta migración. }\end{array}$ \\
\hline
\end{tabular}

Fuente: Elaboración propia con datos facilitados por líderes de 12 ejidos y técnicos del Instituto Internacional de Recursos Renovables (irri), Bases Populares de Tlaxcala, Proyecto de Desarrollo Rural Integral Vicente Guerrero, A. C., Comercializadoray Productores de Maíz de Tlaxcala, A. C.

Selección de los criterios de diagnóstico e indicadores (paso 3). De acuerdo con los puntos críticos, se establecieron y midieron 15 indicadores; cinco en cada dimensión, social (DS), económica (DE) y ambiental (DA), con la finalidad de darle a la investigación un enfoque tridimensional.

Medición y monitoreo de indicadores (paso 4). A continuación se exponen los resultados obtenidos en cada indicador, contemplándolos como parte de cada uno de los cinco atributos que sugieren Masera et al. (2000, pp. 64-66).

\section{Atributo productividad}

La productividad es la capacidad de un agroecosistema de brindar los bienes y servicios necesarios (Masera et al., 2000, pp. 20-21). En este atributo se propusieron y evaluaron tres indicadores: 1) cantidad de granos y zacate cosechados (DA); 2) relación beneficio/costo (DE), y 3) cantidad de producto/energía fósil empleada (DA) (véase el cuadro 2). 


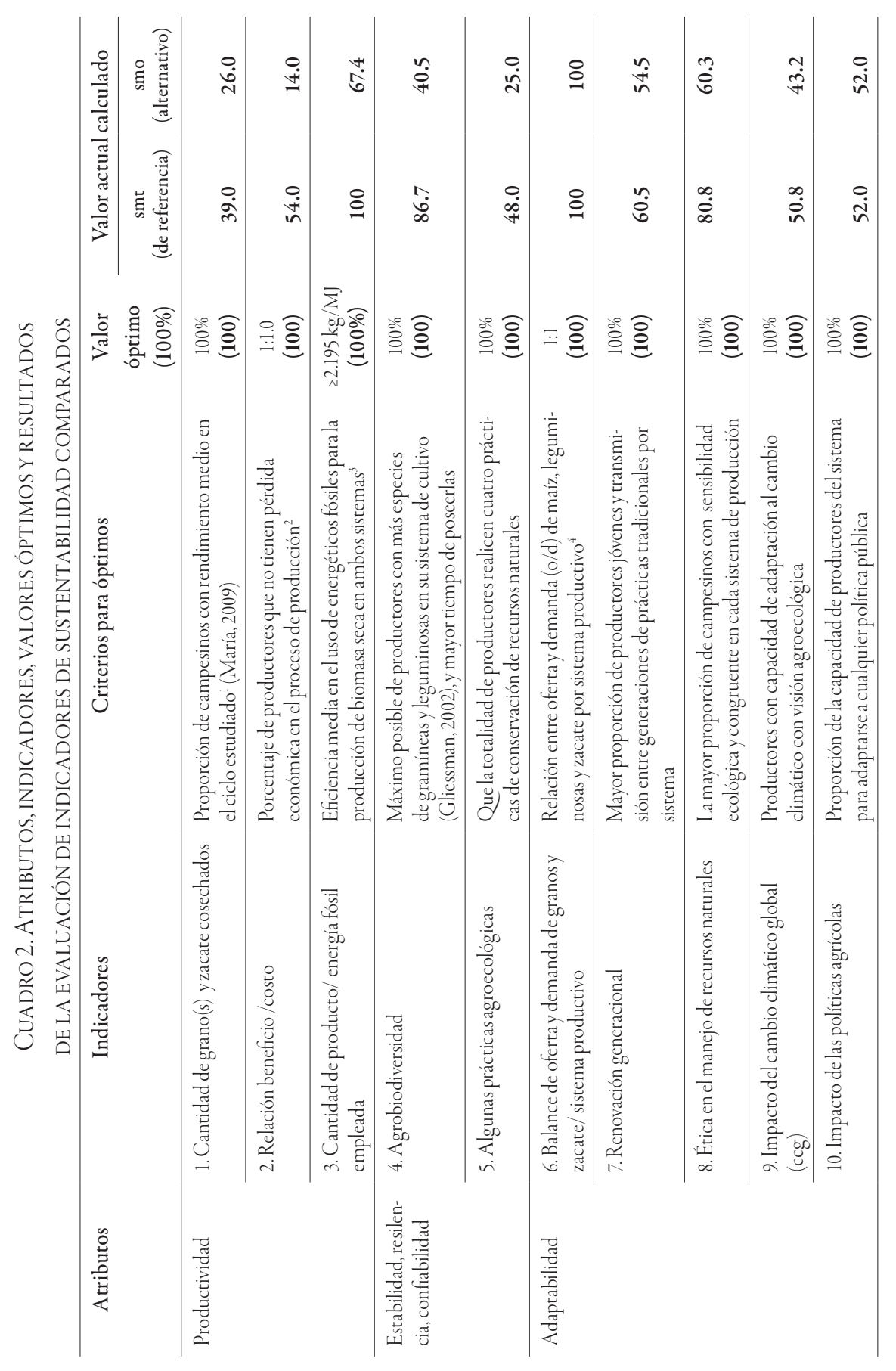




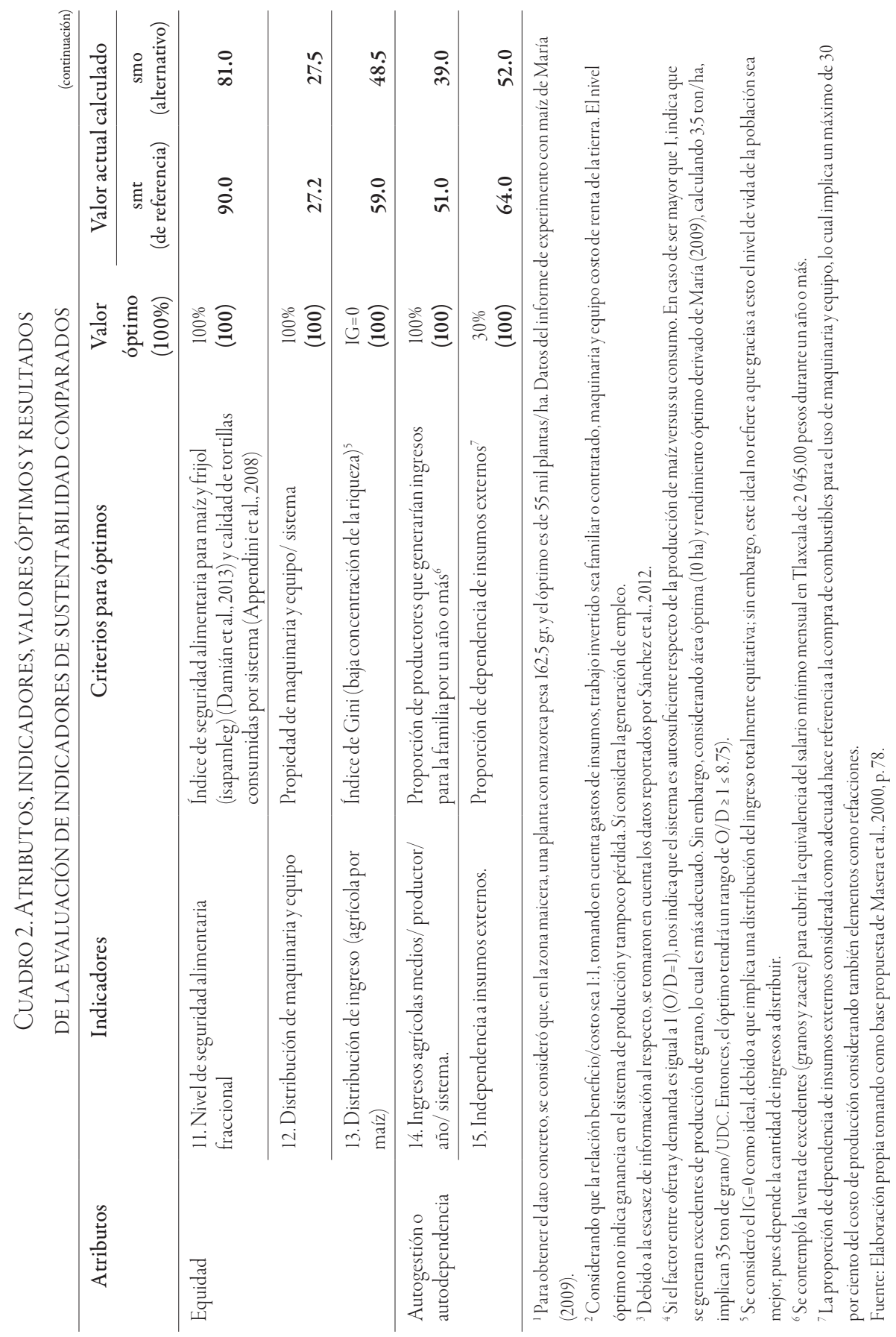


Los resultados de la evaluación favorecen el SMT en los tres indicadores. En el primero, se clasificó el total de productores de cada sistema tomando como referencia el dato que propone María (2009) (nivel óptimo) de $8938 \mathrm{Kg}$ ha-1 (sumando zacate y grano). Con ese rendimiento, se calculó la proporción para cada productor. En esta caracterización, los campesinos que recolectaron menos de 33.3 por ciento se consideraron en el nivel bajo, los que cosecharon de 33.4 a 66.6 por ciento se ubicaron en el nivel medio y los que lograron más de 66.6 por ciento se clasificaron en el nivel alto. Para este indicador, cantidad de granos y zacate cosechados, 39 por ciento de los productores del SMT se ubicó en el nivel alto, y del SMo solo 26 por ciento de los campesinos se situó en esta posición.

La productividad calculada en este trabajo es similar a la reportada por el SIAP (2014) respecto de la producción de maíz (1 $896 \mathrm{Kg}$ ha-1), aunque en el SMo es ligeramente mayor el rendimiento. Sin embargo, al estimar el zacate cosechado (y el frijol, para el caso del SMT), la biomasa seca cosechada es mayor en el sistema de referencia. Este dato coincide proporcionalmente con lo reportado por Astier et al. (2003, pp. 41-42) en la comparación realizada entre el sistema tradicional y el sistema tradicional diversificado, cuyos resultados muestran que donde se diversifica el cultivo del maíz aumenta la disponibilidad de biomasa cosechada.

En el caso del indicador relación beneficio/costo (B:C), también se hicieron cálculos para cada productor, y se consideró adecuada, como producto de esta relación, la unidad, debido a que no hay pérdida ni ganancia; cuando menos, se genera trabajo y se recupera el capital y el trabajo invertidos. Los valores menores de uno representan pérdida y los mayores significan ganancia. En el SMT, 54 por ciento de los productores obtuvo valores mayores a uno, en comparación con el SMo, que solo 14 por ciento logro igual monto.

La relación beneficio-costo depende del costo de los insumos de acuerdo con la oferta y la demanda, así como del precio de venta de la cosecha. Ayala et al. (2013, pp. 389-392) reportan costos de producción en Tulancingo, Hidalgo, de 2282 a 6366 pesos TM-1, lo cual implica utilidades de 1067 pesos como máximo, y pérdidas en otros casos. Para el presente trabajo, la situación es parecida respecto del SMo, pero es diferente para el SMT, debido a que las leguminosas y cucurbitáceas tuvieron mejor precio de venta en el mercado.

Así también, para el indicador cantidad de producto/energía fósil empleada, se valoró el caso de cada productor en cuanto a la frecuencia de uso de tractores y otras maquinarias agrícolas para la preparación de los suelos (barbecho, rastra), de siembra (surcado y siembra), labores culturales (escarda, labra, segunda), cosecha 
(con máquina combinada y uso de desgranadoras, empacado y molido de zacate) y el transporte (flete de cosecha) desde el terreno de cultivo hasta sus respectivas viviendas. Se consideró el óptimo de acuerdo con estudios de Sánchez et al. (2012, p. 148) del valle de Huamantla, Tlaxcala, cuyos valores son $\geq 2.195$ kilogramos de biomasa seca cosechada por megajulio (MJ) derivado de energéticos fósiles. Para este caso, el SMT obtuvo resultados similares al óptimo, y el SMo, 67.4 por ciento de esa proporción, lo cual indica que en el primero se obtienen 2.206 kilogramos MJ-1 de biomasa seca y en el segundo 1.479 kilogramos MJ-1. La relación entre cantidad de biomasa cosechada y la energía fósil empleada fue mayor en el SMT, lo que indica mayor eficiencia. Además, en Estados Unidos, con el modelo productivista, de acuerdo con Pimentel y Pimentel (2005, pp. 5-7) y Pimentel et al. (cit. en Gliessman, 1990, pp. 305-321), se producen 0.172 kilogramos MJ-1. El empleo de altas cantidades de energéticos fósiles durante todo el proceso de producción explica su "alta productividad".

\section{Atributo estabilidad, resiliencia, confiabilidad}

En este atributo se evaluaron dos indicadores: 4) agrodiversidad (DA), y 5) algunas prácticas agroecológicas (DA) (véase el cuadro 2).

En la valoración de agrodiversidad, el SMT obtuvo el mayor valor en cuanto a diversidad de especies (policultivo), al igual que en el número de variedades. Los resultados muestran que 68 por ciento de productores del sistema de referencia tiene tres o más especies cultivadas; además, 94.9 por ciento tiene una variedad de leguminosa. Por parte del SMo, 55.6 por ciento tiene solo una especie (maíz), y 10.1 por ciento, alguna leguminosa. En lo concerniente al tiempo de propiedad del germoplasma, ambos grupos obtuvieron valores similares (68.0 por ciento de los productores de ambos grupos lleva más de 10 años sembrando el germoplasma que tiene en la actualidad). Los resultados favorecen al SMT, que obtuvo 86.7 en promedio, versus 40.5 del SMo. Para el caso de Michoacán, en la comparación que realizaron Astier et al. (2003, pp. 41-44), en el sistema tradicional hallaron seis especies y variedades, y en el tradicional diversificado, nueve. Para el caso de Tlaxcala, la situación es similar en el SMT. En el SMo, la diversificación es menor, aunque 10 por ciento de los productores de este grupo también tiene cuatro especies y variedades.

Para evaluar el indicador algunas prácticas agroecológicas, se preguntó a los campesinos qué prácticas de conservación realizan y la cantidad de estas. Con 
esta información se ponderó el valor de subindicadores. Ambos sistemas obtuvieron valores bajos en barreras vivas (entre 18.0 y 23.3 por ciento). En el SMT, la proporción de policultivo es más alta (100 por ciento), y la conservación de suelos y agua (CSA) (zanjas y bordos) fue mayor que en el SMo (39.5 y 25.0 por ciento, respectivamente).

Los valores calculados para el quinto indicador fueron de 47.8 para el SMT y de 25.0 para el SMo. Al respecto, Gliessman (2002, pp. 301-318) menciona que la agricultura sostenible se basa en la preservación de la calidad de los recursos naturales, y la aplicación de prácticas agroecológicas tiene como objetivo central la producción de alimentos por medio de la conservación de los recursos de los que depende la agricultura.

\section{Atributo adaptabilidad}

La adaptabilidad (flexibilidad) es la capacidad del sistema para encontrar nuevos niveles de equilibrio ante cambios socioambientales en el largo plazo (Masera y López Ridaura, 2000, p. 121). En este atributo se analizaron cinco indicadores: 6) balance de oferta-demanda de granos y zacate/sistema (DE); 7) renovación generacional (DS); 8) ética en el manejo de recursos naturales (DS); 9) impacto del cambio climático (DS), y 10) impacto de las políticas agrícolas (DS).

Para evaluar el indicador balance de oferta-demanda de granos y zacate/sistema, se contemplaron datos por productor respecto de la cantidad de maíz y frijol que consumen semanalmente sus familias (2.66 kilogramos de maíz y 0.26 kilogramos de frijol por persona en promedio ${ }^{1}$ y que son cercanos a lo calculado por el CEDRSSA (2014), que estima 110 kilogramos de maíz requeridos al año por cada mexicano. En el caso de frijol, la Secretaría de Economía (SE, 2015) calculó 11 kilogramos para el consumo per cápita. Esos datos se sumaron al cálculo de maíz requerido para el ganado, de acuerdo con las especies y cantidad de animales que poseen. También se estimó la cantidad total de maíz (grano), leguminosas y zacate que cada campesino produce.

Con esos datos, se valoró la relación oferta/demanda $\left(\mathrm{O}: \mathrm{D}_{\text {parcial }}\right)$ por año y, a la vez, por sistema. Para el maíz, resultó en una proporción de 3.4:1 en el SMT y de 6.1:1 para el SMo. Respecto del frijol, la O: $\mathrm{D}_{\text {parcial }}$ en el SMT fue de 8.1:1, en comparación con el SMo $(0.04: 1)$. En cuanto al zacate, la situación fue contraria, debido

1 Estos datos fueron proporcionados por los productores encuestados. 
a que el SMT muestra un déficit de 20 por ciento de su requerimiento (0.8:1), y el SMo tiene excedente de 40 por ciento al resultar una relación $\mathrm{O}: \mathrm{D}_{\text {parcial }}$ de 1.4:1. Al promediar los tres datos parciales, el SMT obtuvo O: $\mathrm{D}_{\text {total }}=4: 1$, comparado con 2.6:1 calculado para el SMo. No obstante, ambos tienen la capacidad para cubrir con sus excedentes las deficiencias propias, por lo que pueden lograr su autoabasto al 100 por ciento. Así, ambos alcanzaron el nivel óptimo. En el caso que reportan Astier et al. (2003, p. 42), se calcula que se necesitan 4.4 hectáreas para equilibrar la capacidad de carga animal. En el caso de Tlaxcala, se consideró la cantidad de ganado por productor y se calculó la cantidad que requieren de forraje por año y por sistema. Además, para Tlaxcala se conjuntaron los otros elementos citados.

En el indicador renovación generacional, se calculó, del total de personas involucradas en actividades agrícolas, las que tienen edad igual o menor a 35 años. Asimismo se consideró la forma de transmisión intergeneracional de prácticas agrícolas. Los resultados mostraron que en el SMT, 44 por ciento de las personas involucradas en estas actividades son menores a 35 años de edad, y en 77 por ciento de los casos sus familiares les enseñaron las técnicas tradicionales del cultivo del sistema milpa. Respecto de la edad de los productores del SMo, 39 por ciento es menor de 35 años, y 70 por ciento aprendió de su propia familia las técnicas de cultivo que aplica. Los valores medios calculados son 60.5 a favor del SMT y 54.5 en el SMo.

Con estos resultados, se evidencia que la edad media de los propietarios de las tierras es de alrededor de 60 años. Además, en ambos grupos se transmiten los conocimientos empíricos de generación en generación. Lo anterior coincide con lo referido por Hernández Xolocotzi en el momento que caracterizó "la agricultura de huarache” (Hernándezy Aguirre, 1998, pp. 105-106). Además, la edad en ambos grupos muestra la situación de envejecimiento del campo mexicano, que Bartra (2012) deja ver cuando afirma que 50 por ciento de los ejidatarios tiene más de 55 años.

En cuanto a la evaluación del indicador ética en el manejo de recursos naturales, se generó información de la percepción de los productores sobre sus tierras, el agua de temporal que captan para la agricultura y la preservación de sus especies y variedades nativas. Posteriormente se contrastó con la generación de datos sobre lo que cada campesino hace para aprovechar esos recursos. Los resultados favorecieron al SMT, en el que hubo más congruencia entre el discurso y los hechos que en el SMo. En el primero, 25.4 por ciento de los productores no realiza acciones para conservar sus suelos agrícolas, y en el SMo casi la mitad (49.1 por ciento) tampoco realizan obras de CSA, lo cual implica que proporciones similares desaprovechan una parte del agua de las lluvias y sus terrenos están expuestos a la erosión. 
Para la conservación del germoplasma, en el SMT 80.8 por ciento realiza prácticas de selección de semillas, que se guardan para los ciclos siguientes; mientras que en el SMo lo hacen 60.3 por ciento de los campesinos. Aunado a lo anterior, 30 por ciento externaron haber "perdido" alguna variedad o especie de semillas (de frijol, ayocote, haba o maíz). Con las ponderaciones correspondientes, el SMT obtuvo el valor de 80.8 en comparación a 60.3 del SMo. Ambos aspectos evaluados son importantes: en el caso de la agrodiversidad en un país como México, es de gran relevancia por la relación íntima que esta tiene con la diversidad cultural (Toledo y Barrera Bassols, 2008, pp. 15-25, 101-104), ya que da identidad como territorio-nación.

De acuerdo con González et al. (2007, pp. 183-201), los campesinos crean estrategias de supervivencia que se van adecuando al tiempo, a las condiciones biofísicas y socioeconómicas (Chayanov, 1986, pp. 3-5). En este trabajo se plantearon los indicadores impacto del cambio climático (CC) e impacto de las políticas agrícolas (IPA), con los que se consideraron elementos de adaptación externados por los campesinos: el primero es la información que conocen al respecto, seguido por la actitud frente a posibles afectaciones, complementada con las acciones que proponen para su mitigación.

Respecto del CC, en el SMT, 64.4 por ciento conoce información sobre el tema, 64.4 por ciento considera que no le afectará y 52.5 por ciento tiene propuestas y acciones. En promedio, 50.8 por ciento considera afrontar las consecuencias del cambio climático. En el SMo, 39.1 por ciento manifestó estar informado sobre el CC, 44.4 por ciento considera que no le afectará este fenómeno y 35 por ciento plantea propuestas de mitigación. La media y la mediana son más bajas para este grupo de campesinos ( 43.2 y 42.5 por ciento, respectivamente) sobre este tema.

En el caso del IPA, existen datos cercanos entre ambos grupos: en el SMT, en promedio 52.1 por ciento de los productores cuenta con información, considera que no le afecta y tiene mecanismos para adaptarse por medio de acciones. En el SMo, el valor medio es de 51.8 por ciento. Ambos son valores regulares. En su estudio comparativo entre agroecosistemas campesinos de maíz y leche en el valle de Toluca, Brunett (2004, pp. 13-14, 164) encontró diversas condiciones tecnológicas y sociales en que los campesinos tienen una alta capacidad de adaptación.

\section{Atributo equidad}

Este atributo se refiere a la capacidad de un sistema para distribuir de manera justa, intra e intergeneracionalmente, beneficios y costos relacionados con el manejo de 
los recursos naturales (Masera et al., 2000, pp. 19-22). En este atributo se plantearon tres indicadores: 11) nivel de seguridad alimentaria fraccional (DS); 12) distribución de maquinaria y equipo (DE), y 13) distribución del ingreso agrícola (DE) (véase el cuadro 2).

Con el fin de valorar el indicador nivel de seguridad alimentaria fraccional, se consideraron dos factores: la proporción de la producción de maíz y frijol (u otra leguminosa) empleada para autoabasto familiar por año en cada sistema, y la calidad de tortillas que consumen las familias de los productores (forma de elaboración y tipo de maíz usado), considerando lo que sugieren Appendini et al. (2008, pp. 103-127; FAO, 2010) de contemplar la disponibilidad y la calidad.

Para calcular este indicador se utilizó la fórmula propuesta por Damián et al. (2013, pp. 162-163) para estimar el índice de seguridad alimentaria (ISA), a la que se le hicieron adecuaciones para calcular el índice parcial para maíz (ISA pam) como se muestra en la siguiente expresión matemática:

$$
\mathrm{ISA}_{\mathrm{pam}}=\frac{\left(\mathrm{R}_{\mathrm{m}}\right)\left(\mathrm{SS}_{\mathrm{m}}\right) / \mathrm{NMF}}{500^{*}}
$$

Donde:

$\mathrm{R}_{\mathrm{m}}=$ Rendimiento en $\mathrm{Kg} /$ ha de maíz.

$S S_{\mathrm{m}}=$ Superficie sembrada de maíz (ha).

NMF $=$ Número de miembros en la familia del productor.

* Factor que considera que la seguridad alimentaria familiar fraccional para maíz $\left(\mathrm{SAF}_{\mathrm{m}}\right)$ se conseguirá cuando cada miembro de la familia disponga de 500 kilogramos de maíz al año (Damián et al., 2013, pp. 159, 163).

Si el valor de $I S A_{\text {pam }}<1$ : no existe $S A F$; pero $s I S A_{\text {pam }} \geq 1$ : se cuenta con $S A F_{m}$.

En el caso de la seguridad fraccional para el consumo de frijol se utilizó una expresión similar, en la que se adecuó el factor de disponibilidad de leguminosas por persona por año en zonas rurales de acuerdo con datos publicados por la Secretaría de Economía (SE, 2015).

$$
\mathrm{ISA}_{\operatorname{leg}}=\frac{\left(\mathrm{R}_{\mathrm{leg}}\right)\left(\mathrm{SS}_{\mathrm{leg}}\right) / \mathrm{NMF}}{20^{*}}
$$




\section{Donde:}

$\mathrm{R}_{\text {leg }}=$ Rendimiento de frijol u otra leguminosa en Kg ha-1.

$S S_{\text {leg }}=$ Superficie sembrada de leguminosa (ha).

$\mathrm{NMF}=$ Número de miembros en la familia del productor.

* Factor que considera que la $\mathrm{SAF}_{\text {leg }}$ se conseguirá cuando cada miembro de la familia disponga de 13 kilogramos de leguminosa al año.

Si el valor de $\mathrm{ISA}_{\text {leg }}<1$ : no existe $\mathrm{SAF}_{\text {leg; }}$ pero $s i \mathrm{ISA}_{\text {leg }} \geq 1$ : se cuenta con $\mathrm{SAF}_{\text {leg. }}$

Para calcular el índice de seguridad fraccional de maíz y frijol o leguminosa

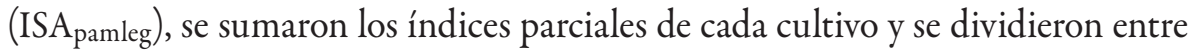
los dos datos como se muestra en la siguiente fórmula:

Los resultados obtenidos mostraron que 64.4 por ciento del SMT satisface el ISA $_{\text {pamleg, }} 22$ por ciento solo satisface el ISA $A_{\text {pam }}$, aunado a 93.0 por ciento de los productores que aún elabora tortillas en casa con maíz criollo principalmente, lo que promedia 89.7 para este grupo. En el SMo, los valores obtenidos son más bajos: 1.6 por ciento satisface el ISA pamleg, 80.3 por ciento satisface el ISA $A_{\text {pam }}$, y 80 por ciento elabora tortillas en casa. El valor medio fue de 80.9 para este conjunto.

Damián et al. (2013, p. 157) reportan que en la comunidad de Cohetzala, Puebla, México, solo 15 por ciento de los productores maiceros alcanza la seguridad alimentaria familiar (SAF), situación generalizada en varias regiones del país debido al elevado costo de producción, condiciones climatológicas y deterioro de suelos, entre otros. Para el caso de Tlaxcala, el nivel de SAF es mayor debido a tres factores principalmente: a) el rendimiento medio es cercano a 2 TM ha-1, en comparación con Cohetzala, donde es de $746 \mathrm{Kh}$ ha-1; b) en el SMo, los productores siembran en promedio 5.5 hectáreas, y c) los productores del SMT siembran maíz, frijol (u otra leguminosa) y calabaza en algunos casos.

Para evaluar el indicador distribución de maquinaria y equipo, se generaron datos respecto de la maquinaria que se emplea para cada labor y si es propia o rentada. Los resultados medios son similares para ambos sistemas de producción, con variaciones en algunos rubros: en el SMT, siete por ciento de los campesinos tiene tractor; 37 por ciento, sembradora (sea de tracción animal o de tractor); 15 por ciento, picadora para zacate o molino; 34 por ciento, bomba para fumigar (principalmente manual); 59 por ciento conserva su yunta, y 11 por ciento tiene desgranadora (de motor eléctrico y a gasolina). En promedio, 27 por ciento tiene maquinaria y equipo. En el grupo de campesinos del SMo, 19 por ciento tiene tractor; 
25 por ciento, sembradora; nueve por ciento, picadora para zacate o molino; 67 por ciento, bomba para fumigar; 36 por ciento, yunta, y nueve por ciento, desgranadora. En promedio, 27.5 por ciento tiene maquinaria y equipo.

Los datos promedio en cuanto a la propiedad de tractores en Tlaxcala son ligeramente mayores (13 por ciento) que los reportados por Damián et al. (2013, p. 171) para Cohetzala (10 por ciento); aunque por grupo, en el SMo 19 por ciento de los productores tiene tractor propio. Respecto de la posesión de yunta, la situación es inversa: mientras en Cohetzala 70 por ciento de los campesinos tiene yunta propia, en Tlaxcala el promedio es de 47.5 por ciento.

Para el indicador distribución del ingreso agrícola se calculó el índice de Gini (IG), que mide la distribución del ingreso. Cuando el valor de IG es cercano a cero indica la existencia de distribución equitativa, y en caso de acercarse a uno muestra que el ingreso se concentra en pocas personas (González, 2009).

Para calcular el índice de Gini se empleó la siguiente fórmula:

$$
\mathrm{IG}=\frac{}{\Sigma}
$$

Donde:

$\mathrm{Fi}$ = frecuencias relativas acumuladas de la población. En este caso, de las muestras en cada sistema.

$\mathrm{Yi}=$ ingreso relativo acumulado de las muestras en cada sistema.

$\mathrm{fi}=$ frecuencias relativas de la muestra.

Con los datos de frecuencias relativas de población $(\mathrm{Fi})$ y de ingresos relativos acumulados (Yi) se elaboró una gráfica de Lorenz para cada grupo.

Los resultados para el SMT, IG=0.408, y para el $S M o, I G=0.516$, indican una distribución más equitativa de ingresos agrícolas, que favorece al SMT. Además, de acuerdo con la curva de Lorenz, en el SMT, 80 por ciento de los campesinos concentra 56 por ciento de los ingresos; en el SMo, 80 por ciento de los productores concentra 44 por ciento de los ingresos. Para el caso de la comparación hecha por Astier et al. (2003, p. 46), los resultados respecto de la distribución de beneficios favorecieron al sistema tradicional diversificado, que a la vez registró mayores ingresos que el sistema tradicional. En este trabajo, la situación es similar: el SMT tiene mejores ingresos y distribución que el SMo. 


\section{Atributo autodependencia (autogestión)}

La autodependencia es la capacidad del sistema para regular y controlar sus interacciones con el exterior, incluidos los procesos de organización y los mecanismos del sistema socioambiental (Masera et al., 2000, pp. 24-25). En términos sociales, se emplea el concepto autogestión, que implica la participación de todos los integrantes de una organización o comunidad en la propiedad y el control de su misma organización (Adizes, 1977, p. 26). Para este atributo se evaluaron dos indicadores: 14) ingresos agrícolas medios/productor/año/sistema, y 15) independencia a insumos externos (semillas, pesticidas, maquinaria y equipo).

En la valoración del indicador ingresos agrícolas medios/productor/año/sistema, se calculó la cantidad necesaria de producción para el autoabasto de acuerdo con los integrantes de cada familia, y los excedentes (granos y forrajes) se valoraron considerando precios medios de venta. Después se dividió el ingreso entre el salario mínimo mensual ( 2045 pesos) a fin de saber cuánto tiempo se podría mantener cada familia con un salario mínimo. En el SMT, 51 por ciento de los campesinos podría tener este ingreso más de un año; 17 por ciento lo conservaría de seis a 12 meses; 25 por ciento, de uno a seis meses, y siete por ciento, ningún mes. En el caso del SMo, 39 por ciento de los productores podría ingresar un salario mínimo durante más de un año; 21 por ciento, de medio año a un año; 30 por ciento, de uno a seis meses, y 10 por ciento, ningún mes.

En los trabajos de Damián et al. (2013, pp. 171-173), todos los campesinos entrevistados se ubican bajo la línea de la pobreza. En Tlaxcala, en el mismo caso, de acuerdo con el CONEVAL (2010), se encuentra 55 por ciento del total de los productores entrevistados. Sin embargo, es mayor la proporción en el SMo (61 por ciento), debido a que no se tienen excedentes suficientes para la venta.

Finalmente, para la evaluación del indicador independencia a insumos externos, se valoró la dependencia por cada productor. Los resultados son los siguientes: en el SMT, tres de cada cien campesinos emplean semillas híbridas, 91 utilizan fertilizantes, 11 aplican herbicidas, 12 emplean insecticidas y 57 hacen uso de maquinaria y equipo para la preparación de suelos, las labores culturales y la cosecha. En promedio, la independencia en cuanto a este tipo de insumos es de 64 por ciento en este sistema de producción. En el SMo, seis de cada cien agricultores emplean semillas híbridas, 93 aplican fertilizantes, 68 emplean herbicidas, 13 usan insecticidas y 61 utilizan maquinaria y equipo. En promedio, la independencia con respecto de este tipo de insumos es de 52 por ciento. Los datos aquí calculados para el SMo se encuentran cercanos a los reportados por el SIAP (2013) para Tlaxcala. 
Los resultados del SMT son diferentes, porque debido a la asociación de cultivos no es posible el uso del paquete tecnológico de la RV.

\section{Integración de resultados (paso 5)}

Para completar el ejercicio de evaluación de indicadores, se planteó un nivel óptimo para cada uno de ellos, que sirvió como punto de referencia en un escenario ideal, en el cual no se forzó al sistema para producir más de su capacidad y tampoco se desperdician las cualidades de este. Se hicieron ponderaciones de acuerdo con los resultados en la valoración de los diversos indicadores. En el cuadro 2 se observa la concentración de los 15 indicadores, sus criterios de óptimos y los resultados para el caso de cada sistema de manejo comparado.

Al considerar los niveles de sustentabilidad según los resultados de cada indicador, en el SMT, uno se ubicó en un nivel bajo de sustentabilidad (distribución de maquinaria y equipo); nueve, en sustentabilidad media, y cinco, en sustentabilidad alta. Por parte del SMo, se ubicaron cuatro indicadores en el nivel bajo de la sustentabilidad: a) cantidad de granos y zacate cosechados; b) relación beneficio-costo;

\section{Figura 1. EVALUACIÓN DE INDICADORES DE SUSTENTABILIDAD}

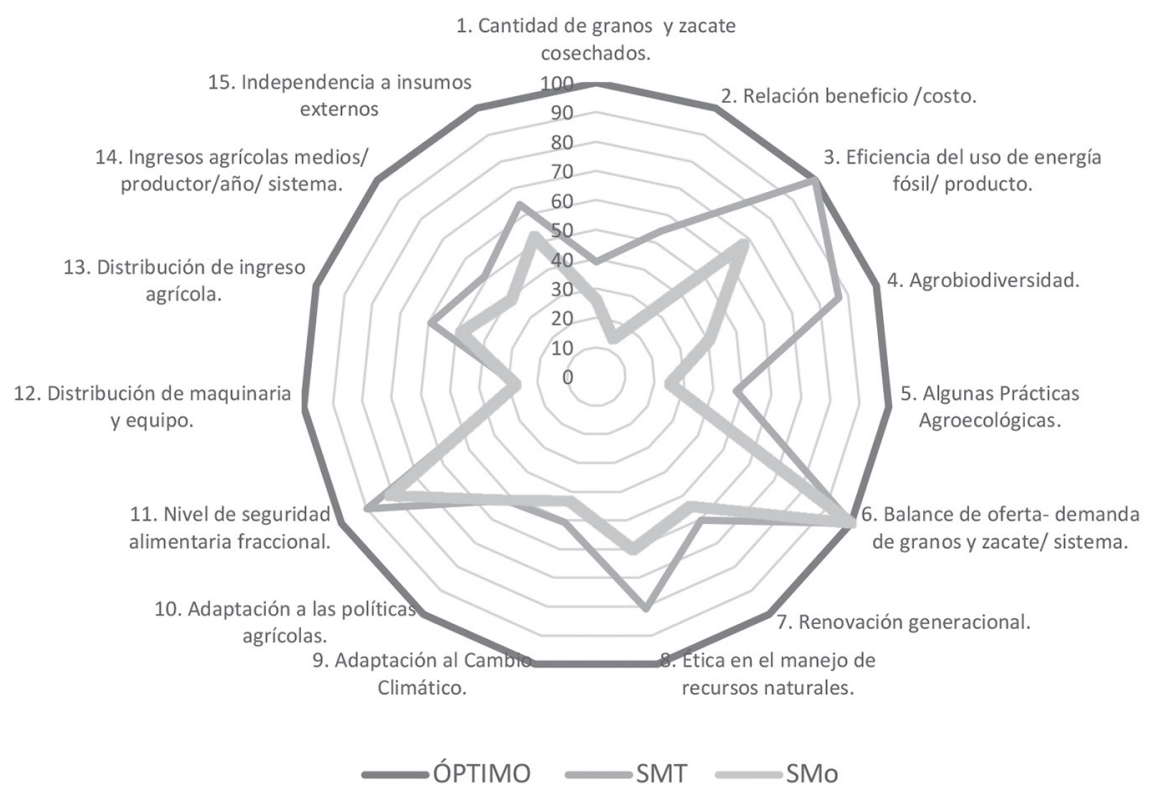


c) prácticas agroecológicas, y d) distribución de maquinaria y equipo. Además, ocho indicadores están en el nivel medio de sustentabilidad y tres están el nivel alto.

Una vez generados los datos con la evaluación de indicadores, el siguiente paso sugerido por Masera et al. (2000, pp. 75-77) es conjuntar los resultados en un gráfico tipo amoeba o amiba, que permite observar el panorama general entre los sistemas de producción comparados (véase la figura 1). Para este caso, se muestra el gráfico con los resultados de 15 indicadores evaluados para cada sistema de manejo.

\section{Conclusiones}

En el balance de los resultados de la evaluación de sustentabilidad se observa que el SMT tiene más indicadores en el área de fortalezas (cinco indicadores) que en el área de debilidades (uno). Por el contrario, en el SMo hay más debilidades (cuatro indicadores) que fortalezas (tres).

Así, se evidencia que el SMT tiene varias ventajas en comparación con la siembra de maíz en monocultivo, porque se produce una diversidad de alimentos, se aprovecha mejor el espacio cultivado, existe una complementariedad entre las especies, es menor el uso y la dependencia de insumos agroquímicos y maquinaria, al igual que el impacto negativo al ambiente. Del mismo modo, este sistema ayuda más a conservar la agrodiversidad.

Con estas evidencias, se concluye que el sistema milpa tradicional es más sustentable que el sistema de maíz en monocultivo porque tiene mayores beneficios para la producción de alimentos, cumple las expectativas de seguridad alimentaria respecto del maíz y las leguminosas, se aprovechan de mejor manera los recursos naturales locales, genera trabajo para las familias campesinas, es de baja entropía y es amigable con el ambiente.

Sin embargo, en ambos sistemas hace falta que los campesinos e indígenas integren otros elementos que hagan sus sistemas productivos más redituables económicamente y, en general, más sustentables. Uno de estos puede ser la introducción de frutales de buena calidad, con aceptación en el mercado. Estas acciones tienen diversas implicaciones: capacitación técnica, inversión de capital que el Estado puede financiar con créditos blandos y facilitación para introducir las primeras cosechas a las cadenas mercantiles.

Respecto del aspecto técnico, es posible emplear técnicas agroecológicas para el mejoramiento de la fertilidad de los suelos, como la elaboración de abonos orgánicos 
(composta, lombricomposta, bioles, etcétera), la implementación de abonos verdes, microorganismos para mejoramiento de la fertilidad (Azospirillum, Rhizhobium), o el uso de microorganismos antagónicos para el control de insectos y enfermedades (Beauveria bassiana, Metarhizium, etcétera), entre otros.

No obstante, el papel del Estado es fundamental para hacer conciencia de la problemática que afronta el agro tlaxcalteca, en particular: altos índices de erosión y deterioro de suelos, elevada contaminación por uso de agrotóxicos, alta dependencia de insumos externos, encarecimiento de insumos y combustibles, desequilibrio ecológico y su consecuente incidencia de insectos y enfermedades, riesgo de erosión genética de semillas nativas, etcétera. Ante este escenario, instancias como la Secretaría de Fomento Agropecuario de Tlaxcala (SEFOA), el programa PESA-FAO, que aplica en esta entidad, y la Cruzada Nacional Contra el Hambre, de la Secretaría de Desarrollo Social, deben destinar recursos para implementar mejoras encaminadas a una agricultura sustentable en términos prácticos, y no solo demagógicos.

Además, es necesaria una campaña permanente que concientice a los consumidores sobre el origen y la forma de producción de los alimentos que llevan a su mesa, para que a la vez exijan que instancias gubernamentales y universidades se comprometan más en mejorar y diseminar técnicas de producción sustentable.

Para evaluar el estado de la sustentabilidad de un agroecosistema, el MESMIS es una herramienta metodológica que se centra en ese objetivo, es sencillo de aplicar, metódico y con resultados acercados a la realidad. Sin embargo, algunas limitaciones encontradas radican en la complicación para incluir subindicadores, así como para encontrar niveles óptimos en cada indicador y para estandarizar formas de medición de factores sociales, culturales y ambientales entre zonas o regiones diversas que son diferentes.

\section{Agradecimientos}

El primer autor agradece especialmente a productoras/es del estado de Tlaxcala por compartir la información de sus sistemas de producción para el presente trabajo. Así también, los más sinceros agradecimientos al Consejo Nacional de Ciencia y Tecnología (CONACYT) de México por el financiamiento y a la Benemérita Universidad Autónoma de Puebla (BUAP) por la facilitación para el mismo. 


\section{BibLIOgRAFÍA}

Adizes, I. (1977). Autogestión: La práctica yugoslava. Distrito Federal, México: Fondo de Cultura Económica.

Altieri, M. A. (1987). The Scientific Basis of Alternative Agriculture. Boulder, Colorado, Estados Unidos: Westview Press.

Altieri, M. A.; Nicholls, C. I. (2000). Agroecología. Teoría y práctica para una agricultura sustentable. Distrito Federal, México: Programa de las Naciones Unidas para el Medio Ambiente.

Appendini, K.; Cortés, L., y Díaz, H. V. (2008). Estrategias de seguridad alimentaria en los hogares campesinos: La importancia de la calidad del maíz y la tortilla. En K. Appendini y G. Torres Mazuera (coordinadores). ¿Ruralidad sin agricultura? Perspectivas multidisciplinarias de una realidad fragmentada (pp. 103-127). Distrito Federal, México: El Colegio de México.

Astier, M.; Pérez Agis, E.; Ortiz, T., y Mota, F. (2003). Sustentabilidad de sistemas campesinos de maíz después de cinco años: El segundo ciclo de evaluación MESMIS. Leisa, Revista de Agroecología, 19(10): 39-46. Recuperado de http:// leisa-al.org/web/index.php/statistics/volumen-19-numero-0/2219-sustentabilidad-de-sistemas-campesinos-de-maiz-despues-de-cinco-anos-el-segundo-ciclode-evaluacion-mesmis

Ayala, G. A. V.; Schwentesius, R. R.; De la O Olán, M.; Preciado, R. P.; Almaguer, V. G., y Rivas, V. P. (2013). Análisis de rentabilidad de la producción de maíz en la región de Tulancingo, Hidalgo, México. Agricultura, Sociedady Desarrollo, 10(4): 381-395. DOI: http://dx.doi.org/10.22231/asyd.v10i4.132.

Azorín, F. (1969). Curso de muestreo y aplicaciones. Madrid, España: Aguilar.

Bartra, A. (2012). Los nuevos herederos de Zapata. Campesinos en movimiento, 19202012. Distrito Federal, México: Coordinadora Nacional Plan de Ayala, Partido de la Revolución Democrática, Circo Maya.

Brunett, P. L. (2004). Contribución a la evaluación de la sustentabilidad. Estudio de caso: Dos agroecosistemas campesinos de maiz y leche del Valle de Toluca (tesis de Doctorado). Universidad Nacional Autónoma de México. Distrito Federal, México.

CEDRSSA (Centro de Estudios para el Desarrollo Rural Sustentable y la Soberanía Alimentaria) (2014). Reporte del CEDRSSA. Consumo, distribución y producción de alimentos: El caso del complejo maiz-tortilla. Distrito Federal, México: Cámara de Diputados, LXII Legislatura. 
Chayanov, A. V. (1986). The Theory of Peasant Economy. Madison, Wisconsin, Estados Unidos: The University of Wisconsin Press.

Cochran, G. W. (1974). Técnicas de muestreo. Distrito Federal, México: Compañía Editorial Continental.

CONEVAL (Consejo Nacional de Evaluación de la Política de Desarrollo Social) (2010). La pobrezapor ingresos en México. Distrito Federal, México: Consejo Nacional de Evaluación de la Política de Desarrollo Social.

Damián, H. M. A.; Cruz, L. A.; Ramírez, V. B.; Romero, A. O.; Moreno, L. S., y Reyes, M. L. (2013). Maíz, alimentación y productividad: Modelo tecnológico para productores de temporal. Agricultura, Sociedad y Desarrollo, 10(2): 157-176. Recuperado de http://www.scielo.org.mx/pdf/asd/v10n2/v10n2a2.pdf.

De Schutter, O. (2012). El derecho a la alimentación. Informe de Misión a México. Relator Especial de la ONU sobre el derecho a la alimentación. Distrito Federal, México: Oficina del Alto Comisionado de las Naciones Unidas para los Derechos Humanos. Recuperado de http://www.hchr.org.mx/images/doc_pub/informe_alimentacin_web.pdf.

FAO (Food and Agriculture Organization of the United Nations) (2009). Proceedings of the Second International Forum on Globally Important Agricultural Heritage System (GIAHS). Theme: Cherishing our Agricultural Heritage Systems for Climate Change Adaptation and Mitigation. Buenos Aires, Argentina: Food and Agriculture Organization of the United Nations.

FAO (Food and Agriculture Organization of the United Nations) (2010). Special Programme for Food Security. Recuperado de http://www.fao.org/spfs/index_es.aspv.

Gliessman, S. (2002). Agroecología. Procesosecológicos en agricultura sostenible. Turrialba, Costa Rica: Impresiones LITOLAT.

González, B. (2009). Cálculo del índice de Gini. Sin pie de imprenta. Recuperado de http://issuu.com/byrong/docs/c_lculo_del_indice_de_gini\#print.

GonzÁlez, J. A.; Del Amo, R. S., y Guirri, G. F. D. (coords.). (2007). Los nuevos caminos de la agricultura: Procesos de conversión y perspectivas. Distrito Federal, México: PROAFT, Universidad Iberoamericana, Plaza y Valdés Editores.

GonzÁlez, M. A., y Ávila, C. J. F. (2014). El maíz en Estados Unidos y en México: Hegemonía en la producción de un cultivo. Argumentos, 27(75): 215-237. Recuperado de http://www.scielo.org.mx/pdf/argu/v27n75/v27n75a11.pdf.

Hecht, S. B. (1999). La evolución del pensamiento agroecológico. En M. A. Altieri (coord.). Agroecología. Bases cientificas para una agricultura sustentable (pp. 15-30). 
Montevideo, Uruguay: Editorial Nordan-Comunidad. Recuperado de https:// www.socla.co/wp-content/uploads/2014/AgroecologiadeALTIERI.pdf?iv=197. Hernández, S. R.; Fernández, C. C., y Baptista, L. P. (2010). Metodología de la investigación. Distrito Federal, México: Mc Graw Hill.

Hernández, X. E., y Aguirre, R. J. R. (1998). Etnobotánica y agricultura tradicional. En L. M. A. Díaz y L. A. Cruz (coords.). Nueve mil años de agricultura en México. Homenaje a Efrain Hernández Xolocotzi (pp. 104-109). Distrito Federal, México: Grupo de Estudios Ambientales, Universidad Autónoma Chapingo.

Hewitt, A. C. (1985).La modernización de la agricultura mexicana, 1949-1970. Distrito Federal, México: Siglo XXI Editores.

INE (Instituto Nacional de Ecología) (1995). Estudio de pais: México ante el cambio climático. Informe técnico México-U.S. Distrito Federal, México: Universidad Nacional Autónoma de México, Centro de Ciencias de la Atmósfera, Country Studies Program Support for Climate Change Studies.

INEGI (Instituto Nacional de Estadística, Geografía e Informática) (2012). Anuario estadístico del estado de Tlaxcala. Aguascalientes, Aguascalientes, México: Instituto Nacional de Estadística, Geografía e Informática.

INEGI (Instituto Nacional de Estadística y Geografía) (2015). Mapa digital de México. Distrito Federal, México: Instituto Nacional de Estadística y Geografía. Recuperado de http://gaia.inegi.org.mx/mdm6/?v=bGF0OjE5LjQzMDAwLGxvbjotOTkuMTMwMDAsejo5LGw6YzExMXNlcnZpY2lvc3x0YzExMXNlcnZp Y2lvcw $==$.

INEGI (Instituto Nacional de Estadística y Geografía) (2016). Panorama sociodemográfico de México 2015. Tlaxcala. Recuperado de http://www3.inegi.org.mx/ sistemas/Panorama2015/Web/Contenido.aspx\#Tlaxcala29000.

María, R. A. (2009). Apuntes de experimentos en INIFAP. Archivos inéditos. Tlaxcala de Xicohténcatl, Tlaxcala, México.

Masera, O.; Astier, M., y López Ridaura, S. (2000). Sustentabilidad y manejo de recursos naturales: El marco de evaluación MESMIS. Pátzcuaro, Michoacán, México: Grupo Interdisciplinario de Tecnología Rural Apropiada, Mundi-Prensa México, Instituto de Ecología de la Universidad Nacional Autónoma de México.

Masera, O., y López Ridaura, S. (eds.) (2000). Sustentabilidad y sistemas campesinos. Cinco experiencias de evaluación en el México rural. Pátzcuaro, Michoacán, México: Grupo Interdisciplinario de Tecnología Rural Apropiada, Mundi-Prensa México, Universidad Nacional Autónoma de México, Programa Universitario de Medio Ambiente. 
Max Neef, M. A.; Elizalde, A., y Hoppenhayn, M. (2001). Desarrollo a escala humana. Conceptos, aplicaciones y algunas reflexiones. Barcelona, España: NordanComunidad, Icaria.

Pimentel, D.; Dazhong, W., y Giampietro, M. (1990). Technological Changes in Energy use in U.S. Agricultural production. En S. R. Gliessman (ed.). Agroecology. Researching the Ecological Basis for Sustainable Agriculture (pp. 305-321). Nueva York, Estados Unidos: McGraw-Hill.

Pimentel, D., y Pimentel, M. (2005). Energy Use in Agriculture: An Overview. LEISA Magazine, 1(21): 5-7. Recuperado de http://www.cigrjournal.org/index.php/ Ejounral/article/view/1044/1037.

PROAGRO PRODUCTIVO (2014). Listado preliminar de beneficiarios primavera verano 2014 del estado de Tlaxcala. Distrito Federal, México: Secretaría de Agricultura, Ganadería, Desarrollo Rural, Pesca y Alimentación. Recuperado de http:// www.sagarpa.gob.mx//agricultura/Programas/proagro/Beneficiarios/PV14/ TLAXCALA_PV14.zip.

SAGARPA (Secretaría de Agricultura, Ganadería, Pesca y Alimentación) (2014). Delegación de la SAGARPA en Tlaxcala. Recuperado de http://www.sagarpa. gob.mx/Delegaciones/tlaxcala-gob-estado/Paginas/default.aspx/.

SÁnchez, M. P., y Castro, P. F. (2011). Prácticas agroecológicas para una agricultura sostenible. Tlaxcala de Xicohténcatl, Tlaxcala, México: El Colegio de Tlaxcala, Proyecto de Desarrollo Rural Integral Vicente Guerrero.

SÁnchez, M. P., y Hernández, O. P. (2014). Sistema milpa: Elemento de identidad campesina e indigena. Distrito Federal, México: Programa de Intercambio, Diálogo, Asesoría en Agricultura Sostenible y Seguridad Alimentaria.

Sánchez, M. P.; Ocampo, F. I.; Parra, I. F.; Sánchez, E. J.; María, R. A., y Argumedo, M. A. (2012). Evaluación de la sustentabilidad del agroecosistema maiz en la región de Huamantla, Tlaxcala (tesis de Doctorado). Colegio de Posgraduados en Ciencias Agrícolas Campus Puebla. Puebla, Puebla, México.

SE (Secretaría de Economía) (2015). Delegación de la Secretaría de Economía en Tlaxcala. Recuperado de http://www.2006-2012.economia.gob.mx/delegaciones-de-la-se/ estatales/tlaxcala.

Sevilla, G. E., y González, D. M. (1992). Ecología campesinado e historia. Madrid, España: Ediciones de la Piqueta.

SIAP (Servicio de Información Agroalimentaria y Pesquera) (2013). Tecnificación. Archivos que contienen los cuadros tabulares con datos estadísticos del uso de 
tecnología y servicios en la superficie agrícola. Recuperado de https:/www.gob. $\mathrm{mx} /$ siap/documentos/tecnificacion.

SIAP (Servicio de Información Agroalimentaria y Pesquera) (2014). Cierre de la producción agrícola por estado: Cultivo de maíz en 2014. Anuario estadístico de la producción agrícola. Recuperado de http://infosiap.siap.gob.mx/aagricola_siap_gb/ icultivo/.

Toledo, V. M., y Barrera-Bassols N. (2008). La memoria biocultural. La importancia ecológica de las sabidurias tradicionales. Barcelona, España: Junta de Andalucía, Icara Editorial.

USDA (United States Department of Agriculture) (2014). Adoption of Genetically Engineered Crops in The United States. Recent Trends in GE Adoption. Washington, D. C., Estados Unidos: United States Department of Agriculture. Recuperado de http://www.ers.usda.gov/data-products/adoption-of-genetically-engineeredcrops-in-the-us/recent-trends-in-ge-adoption.aspx.

USDA (United States Department of Agriculture). (2015). Coarse Grains: World Markets and Trade. Washington, D. C., Estados Unidos: United States Department of Agriculture. Recuperado de http://apps.fas.usda.gov/psdonline/circulars/graincorn-coarsegrains.pdf. 